\title{
RESEÑAS/REVIEWS
}



NICOLA POLLONI, Domingo Gundisalvo. Una Introducción, Madrid-Porto, editorial Sindéresis, 2017, 165 páginas.

En este libro Nicola Polloni propone una lectura, a la vez detallada y preambular, del pensador español Domingo Gundisalvo. Polloni es gran conocedor del autor, sobre quien realizó su Tesis Doctoral ('Ontologia divergenti: Uno studio sul sincretismo metafísico di Gundisalvi', Pavia / Bellaterra, 2015; Unpublished doctoral thesis, Glimpses of the Invisible: Doctrines and Sources of Dominicus Gundissalinus' Metaphysics, PIMS, Toronto, forthcoming) y ha escrito numeroso artículos. Esta vez presenta al público a un autor que puede ser considerado como «el primer escritor latino en afrontar los problemas de coherencia implícita entre las tradiciones filosóficas cristiana, islámica y judía, problemas que se desarrollarán y madurarán en el siglo XIII)» (p. 11); pues se trata de un escritor que traduce en el Toledo del XII fuentes árabes, como Avicena, y judías, como Ibn Gabirol, a la vez que repiensa tesis principales del platonismo de Chartres.

En el primer capítulo rastrea las fuentes que proporcionan el marco adecuado en el que transcurrió la vida de Domingo Gundisalvo (1115/1125), archidiaconus Segobiensis apud Toletum; así como la controversia acerca de la existencia y la particular configuración de la denominada «Escuela de traductores de Toledo». Analiza las tesis de Ch. Burnett, J. García Fayós, Ch. Haskins, A. Jourdain, M. Alonso Alonso, D. Jacquard, M. Th. D’Alverny, entre otros. Sostiene la permanencia en Toledo del traductor y filósofo entre 1152 y 1166 , analizando en profundidad la identidad de sus colaboradores: Avendauth y Johannes Hispanus (p. 27). El apartado titulado «El círculo de Gundisalvo» (pp. 36-39), aclara de modo definitivo las traducciones gudisalvianas, basándose en fuentes histórico-especulativas, lo cual ayuda a entender su pensamiento.

El capítulo segundo está dedicado a los «Tratados epistemológicos», cuestión tratada dentro de todo el proyecto filosófico de Gundisalvo. Aclara que los tratados tradicionalmente atribuidos a Gundisalvo son seis: De scientiis, De divisione philosophiae, De immortalitate animae, De anima, De unitate et uno y De processione mundi. Estos escritos, sostiene Polloni, abarcan tres «núcleos temáticos» (p. 44): el psicológico, el epistemológico o gnoseológico y el metafísico-ontológico.

En todo caso, queda claro que el filósofo toledano tiene como fuentes autores del neoplatonismo y del aristotelismo árabe. Dentro del movimiento de las traducciones de Toledo, Polloni destaca la influencia del Didascalion de Hugo de san Víctor. Analiza el De scientiis, y el distinto valor de la lógica en esta obra y en De divisione philosophiae. Esta última obra mencionada, en el análisis de Polloni, sea quizá la que fija claramente la posición filosófica gundisalviana, en cuanto supone un orden del saber, no clasificado tan claramente hasta ahora y donde se deja ver la influencia de Boecio. La relación entre los objetos de las ciencias y los tipos de conocimiento humano, según su relación a la materia, fija un conjunto de ciencias que introduce nuevos tramos del conocimiento (Jolivet, p. 63).

La psicología de Gundisalvo, abordada en el capítulo tercero, retoma los temas de los tratados medievales acerca del alma, señalando claramente las fuentes de Boecio, de Macrobio, Calicidio, Cicerón y san Agustín (p. 74). Se plantea con claridad la cuestión de la sustancialidad del alma, sin ser esta corporal. Afirma con ello la inmortalidad del alma humana, que no tendría dependencia del cuerpo. De ahí se entiende la disgresión final sobre el conocimiento del alma tras su separación del cuerpo (84).

Especialmente interesante resulta el capítulo cuarto y el estudio de la metafísica de gundisalvo, a través del De unitate et uno y el De processione mundi. La cuestión de la unidad, que es investigada en relación con la fuente gabiroliana, afronta bien el tema de lo Uno como tema principal de la filosofía. El De processione mundi mostraría a su vez la lectura gundisalviana de Avicena a través de Ibn Gabirol (110). Ambas obras son presentadas desde la cuestión clave de la causalidad. Sumamente esclarecedor es la indagación sobre la relación entre la unidad creada y la unidad creadora, aquella participada; y, aparejado a ello, la metáfora de la luz como para indicar la luminosidad difusiva del creador sobre el mundo que participa de la Fuente de la luz. De este modo, creación y manifestación de la unidad aparecen 
como temas típicamente gundisalvianos a la hora de tartar la multiplicidad y su referencia a la unidad. El análisis de la processio se muestra semejante en la obra de madurez de Domigo Gundisalvo.

La bibliografía y los estudios recogidos al final del libro resultan sumamente exhaustivos. Con todo ello, podemos decir que, si bien nos hallamos ante una Introducción a la obra y al pensar de Domingo Gundisalvo, el studio realizado por Nicola Polloni resulta fundamental para adentrarse en la filosofía del autor tratado, pero también en los problemas que acechan a la filosofía a finales del siglo XII. «Filósofo de frontera» (165), ofrece claves que no pueden obviarse ante la tan debatida cuestión platonismoaristotelismo en el paso del siglo XII al siglo XIII.

MARÍA JESÚS SOTO-BRUNA

\section{ANDREA FALCON (ed.), Brill's Companion to the Reception of Aristotle in Antiquity. Series: Brill's companions to classical reception. Series Editor Kyriakos N. Demetriou. Vol. 7. Leiden; Boston: Brill, 2016. ISSN 2213-1426.}

This volume contains twenty-three articles concerning the reception of Aristotle's philosophy in Antiquity arranged in three main sections: the first section deals with the Hellenistic reception of Aristotle; the second section is about the post-Hellenistic engagement with Aristotle; as for the third section, it addresses the reception of Aristotle in Late Antiquity. The volume also includes an index of ancient names and one more index containing the passages with the ancient authors and their works.

The wonderful introduction of the editor, Andrea Falcon, justifies the order followed in this volume as well as the proposed periodization. First of all, by pointing out the lines of continuity between the Hellenistic and the post-Hellenistic era. Secondly, Falcon acknowledges the different possible objections concerning the treatment of Late Antiquity as a distinct period in the ancient reception of Aristotle as well as the use of Porphyry to mark the beginning of Late Antiquity -which, as a result, makes of Plotinus the last of the post-Hellenistic philosophers to engage with Aristotle. The author's response is that this has to do with the integration of Aristotle and Plato as the defining element of the reception of Aristotle in Late Antiquity, which justifies the election of Porphyry as its starting point. Thirdly, the introduction also touches on the different documented reactions to the philosophy of Aristotle in antiquity. These reactions include indifference and resistance to Aristotle's philosophy, but also the appropriation of his ideas and doctrines. In this regard, Falcon states that the most common reaction in the post-Hellenistic period was a selective acceptance of Aristotle's philosophy which culminated in the attempt to integrate Aristotle and Plato in one single philosophical position in Late Antiquity, although that search for harmony was implemented in different ways within this period.

Let us now move on to the first section of the volume, which is called The Hellenistic Reception of Aristotle and includes three chapters. In the first one, «Aristotle and the Hellenistic Peripatos: From Theophrastus to Critolaus», David Lefebvre argues against the idea that there was a decline of the Peripatetic school and shows how, in fact, the Hellenistic Peripatos was offering new solutions for a different philosophical context. In this way, we should understand the history of the Hellenistic Peripatos as the aftermath of the earliest reception of Aristotle's philosophy, and thus, as an evolution inside the Peripatetic school, rather than as its decline. «Aristotle and the Garden», by Francesco Verde, is the second chapter in this volume. Verde focuses on the explicit and implicit references to Aristotle in the writings of Epicurus and the Epicureans and illustrates that there was not only a polemical relationship between the philosophers of the Garden and Aristotle, but also a fruitful dialogue between the two philosophical schools which should be understood as dialectical. Given that Epicureans were in dialogue with the great philosophical systems of the fifth and fourth centuries BC, Verde says, «Hellenism marked no real break in the philosophical debate» (p. 51). In the third chapter, «Aristotle and the Stoa», Thomas 
Bénatouil focuses on the explicit references to Aristotle found on the early Stoics, addressing the three parts of Stoic philosophy, namely, physics -where there are fewer explicit references to Aristotle-; logic, and especially dialectic, the similarities between Aristotle and the Stoics can be found mostly in the features or uses of this discipline; and ethics, in which Aristotle's influence was more widespread and where there are underlying conceptual parallels between Aristotle and Stoicism.

The second part of the volume, The Post-Hellenistic Engagement with Aristotle, is sub-divided into two units: The Peripatetic Tradition -five chapters- and Beyond the Peripatetic Tradition -seven chapters-. The first unit starts with Andronicus of Rhodes and finishes with Alexander of Aphrodisias, whereas the second goes from the reception of Aristotle in Antiochus and Cicero to Aristotle in the Aëtian Placita, including authors such as Plotinus and Galenus and the presence of Aristotle in their works.

The Peripatetic Tradition starts with the chapter written by Myrto Hatzimichali, «Andronicus of Rhodes and the Construction of the Aristotelian Corpus». In this article, Hatzimichali explains that Andronicus of Rhodes can only be credited with the activity of canon- or corpus-organisation of the works of Aristotle, but not with that of textual criticism. The fifth chapter, «Aristotelianism in the First Century BC», written by Andrea Falcon, shows that there was not one single nor dominant interpretation of Aristotle in the often referred to as the early commentators, but that there were in fact competing interpretations within the Peripatetic tradition in the first century BC. The sixth chapter, «Peripatetic Ethics in the First Century BC: The Summary of Didymus», by Georgia Tsouni, deals with Didymus and the problems of authorship around him. The article also gives an overview of the structure and content of the Peripatetic doxography as well as of its original Peripatetic sources.

The seventh chapter, «Aristotelianism in the Second Century AD: Before Alexander of Aphrodisias», by Inna Kupreeva, illustrates that Alexander of Aphrodisias' work is a culmination of the revival of Aristotelianism that took place in the second century AD. In order to do this, the author analyses different authors from this period and shows how the commentary increasingly became the way of doing philosophy. The main novelty of this article is that it expounds that Peripatetic authors had a detailed knowledge of the Aristotelian corpus, which has not been documented before (p. 156). Chapter eight, «Alexander of Aphrodisias», by Cristina Cerami, characterises Alexander of Aphrodisias as a turning point with respect to the preceding Peripatetic tradition and suggests the term Neo-Aristotelianism to talk about his philosophical project, since it marks the beginning of a new philosophical agenda.

As for the second unit of The Post-Hellenistic Engagement with Aristotle, that is, Beyond the Peripatetic Tradition, it starts with «The Reception of Aristotle in Antiochus and Cicero», in which John Dillon questions the sources to which Antiochus of Ascalon, and Cicero after him, had access to and how this affected their interpretation of Aristotle. Chapter ten, «The Appropriation of Aristotle in the PsPythagorean Treatises», by Angela Ulacco, shows how the Pythagorean pseudepigrapha appropriated some of Aristotle's doctrines for the Pythagorean-Platonic tradition and that such appropriation had enormous consequences for the later reception of Aristotle in Platonism: philosophers in Late Antiquity would use it in order to demonstrate the essential agreement between Aristotle and Plato, both believed to ultimately have had their source in Pythagoras' thought.

Alexandra Michalewski's «The Reception of Aristotle in Middle Platonism: From Eudorus of Alexandria to Ammonius Saccas», covers the reception of Aristotle in Middle Platonism and shows how the attitudes towards Aristotelian philosophy varied in Middle Platonism, going from an open hostility to the strategic use of some Aristotelian terms. The author also points out that Ammonius Saccas was the one introducing a new approach to Aristotle by attempting to show that Plato and Aristotle were in fact in agreement on their most important theories. Chapter twelve, «Galen's Reception of Aristotle», by R. J. Hankinson, describes Aristotle as an ambivalent figure in Galen's reception of his thought: whereas Galen had a strong connection with Aristotle's methodology, he thought Aristotle was wrong in certain important facts, such as the transmission of sensation and intelligence. Hankinson presents the 
convergences and divergences between Aristotle and Galen in logic and demonstration; physics and metaphysics; physiology and embryology, and psychology.

In chapter thirteen, «Plotinus' Reception of Aristotle», Sara Magrin shows that Plotinus' reception of Aristotle rests mainly on the appropriation of a method for philosophical inquiry, which is that of solving significant aporiai, and that ultimately he thought of this as a refinement of Socratic dialectic. «The Ancient Biographical Tradition on Aristotle», by Tiziano Dorandi aims to answer two questions, for the first one, who was Aristotle, the author points out towards biographies having been written once Aristotle became a notorious philosopher, and how these lives provided an unreliable picture of Aristotle as a historical figure. The second one deals with what remains of these biographical texts written in Antiquity, and points at two main traditions in this regard, one sympathetic, the other hostile towards Aristotle. "Aristotle in the Aëtian Placita», by Jaap Mansfeld, covers the importance that this doxographical treatise had in order to understand the reception of Aristotle's philosophy in Antiquity. Mansfeld also provides an overview of the Placita material referring to Aristotle and of the parts informed by an Aristotelian methodology.

The third and last section of the volume, Aristotle in Late Antiquity, contains eight chapters, going from Porphyry to early Christian philosophers on Aristotle. Riccardo Chiaradonna is the author of the first article within this section, «Porphyry and the Aristotelian Tradition», which shows that Porphyry's specific contribution was «to have brought into the philosophical background of Platonism an in-depth exegesis of Aristotle's treatises and an extensive knowledge not only of Aristotle but also of the Aristotelian commentary tradition» (p.337) and how this exegetical work on Aristotle can be seen as a tacit response to Plotinus. Chapter seventeen, «An Intellective Perspective on Aristotle: Iamblichus the Divine», by Jan Opsomer, points out how Iamblicus applies a new exegetical strategy consisting on taking Aristotle and the Peripatetics to be the heirs of the Pythagorean-Platonic tradition, and thus the similarities among them, since they belong in the same tradition, are to be found by means of a careful analysis of Aristotle's texts. «Themistius», by Arnaud Zucker, draws attention to the Themistian paraphrases as essential within the Aristotelian tradition and as a redefinition of this exegetical method. «Syrianus and Proclus on Aristotle», by Pieter d'Hoine, depicts Syrianus and Proclus as having a critical attitude towards Aristotle and shows how they subordinated Aristotle to Plato rather than attempting to dissolve their apparent contradictions.

The twentieth chapter, «Ammonius and the Alexandrian School», by Michael Griffin, analyses the curricula of the Alexandrian school and the ideas of some of its lecturers, such as Ammonius or Olympiodorus. The article also touches on their relationship to Christianity. Pantelis Golitsis is the author of chapter twenty-one, «Simplicius and Philoponus on the Authority of Aristotle» which presents a detailed account of Simplicius' vindication of Aristotle and how this should be better understood as a response to Philoponus, whose criticism of Aristotle is also a part of this chapter. «Aristoteles Latinus: The Reception of Aristotle in the Latin World», by Christophe Erismann, deals with the particularities of the reception of Aristotle's philosophy in the Latin world, which would have the Organon as its exclusive focus up to the twelfth century. This, together with Porphyry's Isagoge, would determine the nature of the Latin philosophical thought in this period. The last chapter in this compilation, «Early Christian Philosophers on Aristotle», by George Karamanolis, shows that early Christian philosophers had a critical and sometimes hostile attitude towards Aristotle in the 2nd to the 4th centuries AD. How that attitude changes and varies through the centuries in early Christian thinkers, mainly Clement, Origen and the Cappadoceans, is also a subject within this article.

This volume as a whole provides us with a deeper understanding of the role of Aristotelian philosophy in this period and its multi-faceted reception from the Hellenistic era to Late Antiquity. By means of this volume we can also attain a clearer view of the relations between paganism and Christianity in this period and on how some philosophical views entered into conflict with the Christian ones. The articles display rigorous scholarship and cover a wide range of philosophical topics without leaving philological issues 
untreated. In addition, the clarity with which the authors explore the different topics makes of this compilation a useful tool for specialists and non-specialists alike. These reasons make of this work a very good source for understanding philosophy within this period and also the complexity of the reception of Aristotle in Antiquity.

PILAR HERRÁIZ OLIVA Universidad de Murcia, España

\section{PÉREZ-ILZARBE, PALOMA; CEREZO, MARÍA (eds.); History of Logic and Semantics. Studies on the Aristotelian and Terminist Traditions. In Memory of Angel d'Ors, Brill, Leiden, 2017.}

Se trata del libro homenaje dedicado a Angel d'Ors (1951-2012) por sus colaboradores más estrechos. A este respecto Paloma Pérez-Ilzarbe y María Cerezo han reunido trece participaciones que destacan diversos aspectos de su actividad investigadora en la lógica aristotélico-escolástica, ya sea en la Universidad de Navarra o en la Universidad Complutense, donde fue catedrático. Al homenaje se suman numerosos medievalistas de diversas nacionalidades interesados por estos temas, que incluyen la teoría de los términos, las obligaciones y la buena consecuencia lógica, los insolubles y las falacias lógicas, así como el modo discursivo de abordar los problemas metafísicos últimos por parte de los tratados lógicofilosóficos medievales. El libro reúne trece contribuciones:

1) Allan Bäck analiza la génesis de la denominada falacia por accidente, tanto en la lógica clásica como moderna, a partir de las paradojas generadas por la específica atribución "per accidens" de una determinada propiedad, sin que necesariamente se tenga que tratar de una predicación accidental.

2) José Miguel Gambra distingue entre el "ser en" un sujeto y la predicación por accidente, a través de la interpretaciones propuestas por Boecio y Domingo de Soto. Se justifica así la idea de que lo que es en un sujeto se dice por accidente de la sustancia.

3) María Cerezo analiza las dificultades que plantea la teoría del significado en el De Grammatico de Anselmo de Canterbury, y ofrece una interpretación de las distinciones semánticas presentes en el diálogo, a partir de algunas notas escritas dejadas por Ángel d'Ors.

4) Luisa Valente analiza la noción de deseo en las proposiciones incompletas y en las completas noasertivas en la Dialéctica y en los Comentarios al Peri Hermeneias de Abelardo. En su caso prolongó las propuestas de Aristóteles y de Boecio, añadiendo una visión de la lógica como disciplina de la comunicación verbal.

5) Joke Spruyt analiza la noción de buena consecuencia lógica en las Introducciones Montanas mayores, una Guía de estudio de la Escuela lógica dominicana de Santa Genoveva de Alberico de París. Se analiza la separación entre las voces significativas y las no significativas, entre el nombre y el verbo, así como la noción de proposición, de relación lógica y de obligación.

6) Angel d'Ors analiza el papel de la partícula interrogativa latina " $a n$ " en diversos tratados lógicos, en particular en el Ars Burana, en los Sincategoremata Monacensia y en los Sincategoremata de Robert Bacon. Se analizan problemas relativos a la "referencia" de las oraciones, y a las condiciones de verdad o falsedad de las proposiciones construidas con esta partícula.

7) C.H. Kneepkens analiza la colección de 38 sofismas gramaticales contenidos en la biblioteca británica de Londres, en el llamado manuscrito de Burney, cuyo origen se retrotrae el siglo XIII. Se destaca el distinto papel desempeñado por las suposiciones sintácticas y semánticas en la formulación de dichos sofismas. 
8) Mikko Yrjönsuuri compara la noción de obligación y la de buena consecuencia lógica, analizando cómo algunas técnicas de la disputa "Sobre obligaciones" se usan para aclarar distintas nociones de consecuencia.

9) Sten Ebbesen analiza un sofisma británico del siglo XIII, que sirve para investigar el comportamiento lógico de los operadores exclusivos ("solo") y al mismo tiempo algunos problemas específicos de los términos relativos ("padre").

10) Calvin G. Normore analiza la interpretación dada por Angel d'Ors de la regla de buena consecuencia aceptada por Jean Buridan, "A partir de lo imposible cualquier cosa se sigue". Se desarrolla la idea de que la noción de consecuencia lógica no puede ser caracterizada exhaustivamente, sino que equivale al conjunto (incompletable) de reglas de inferencia.

11) E. Jennifer Ashworth analiza un tratado de Oxford del siglo XIV acerca de las obligaciones de Richard Billingham. Analiza una de las reglas de este tipo de disputa lógica, tratando de entender el sentido de las restricciones que la acompañan.

12) Stephen Read analiza un sofisma de Richard Kilvington en relación con la teoría de las obligaciones. Se analiza especialmente el sentido lógico de la denominada inferencia a partir de lo imposible.

13) Paloma Pérez-Ilzarbe analiza el significado de la cópula "es" en los Sincategoramata de Fernando de Enzinas. Se destaca la idea de que la cópula puede significar el tiempo de tres modos distintos (más bien que tres tiempos distintos), según el verbo "ser" se formule en presente, pasado o futuro.

El libro incluye también un breve In Memoriam (pp. vii-x) y la bibliografía completa de Angel d'Ors (pp. xi-xviii). Sin duda las aportaciones de Angel d'Ors a la lógica medieval española y europea están todavía por desarrollar. Especialmente sus contribuciones acerca del papel desempeñado por Pedro Hispano, por Diego Mas, o por Domingo de Soto, así como su contribución al estudio de la historia de la lógica del siglo XX, especialmente a través de sus trabajos sobre Frege. Muchas de estas publicaciones están aún inéditas. Además, todas las participaciones en este libro homenaje ponen de manifiesto su específico interés por el desarrollo de la lógica británica y continental, así como el influjo recíproco que ejercieron en el desarrollo de la lógica en España. Se trata en cualquier caso de un influjo ajeno al interés por Aristóteles demostrado en esta misma época por la filosofía islámica, ya sea a través del averroísmo latino o de otro tipo de influencias. En cualquier caso estamos en presencia de uno de los grandes especialistas en la lógica medieval cuya figura está aún por descubrir, como ahora se pone de manifiesto en este libro homenaje que se le ha dedicado.

CARLOS ORTIZ DE LANDÁZURI Universidad de Navarra

\section{IDEL, MOSHE; Estudios sobre la cábala en Cataluña, Alpha Decay, Barcelona, 2016, 311 pp.}

Moshe Idel, de origen rumano (1947), afincado en Israel desde 1963, ha sido el continuador del pensamiento de Ghershom G. Scholem sobre la mística judía y titular de la cátedra Max Cooper sobre pensamiento judío en la Universidad Hebrea de Jerusalén. La publicación en 1988 de Kabbalak. New Perspectives, por la Princeton University Press, propició un cambio profundo en el acercamiento a una tradición histórica de muy difícil acceso, debido en gran parte al hermetismo tan estricto cultivado en la forma básicamente oral de trasmisión de los conocimientos. Sin embargo fueron los creadores de una tradición talmúdica de muy amplia difusión por los más diferentes pueblos y culturas, con controversias muy enconadas acerca de los motivos filosóficos y teológicos más dispares, que a su vez se remontan a distintos pasajes bíblicos o a producciones propias que ya están incorporadas al acerbo de nuestra civilización occidental. En cualquier caso se trata de un mundo por descubrir donde resulta esencial la 
intervención del erudito para otorgar el significado preciso a un determinado término tratando a su ves de dar continuidad a una controversia aparentemente agotada, pero con prolongaciones en ocasiones un tanto sorprendentes. Se trata, además, de una tradición adaptada a su tiempo y abierta a múltiples influencias, ya sean árabes o cristianas, sin por ello perder su fisonomía propia y con frecuencia recogida a través de los escritos de sus seguidores, pasados ya bastan años desde su formulación inicial. En este caso se trata de reconstruir la tradición cabalística que permitió a lo largo del siglo XIII-XIV la introducción de la mística judía, primero en Cataluña y después en España, a partir de la Provenza francesa. Pero a su vez ejercería sobre esta tradición un fuerte influjo de La guía para perplejos de Maimónides o Averroes (siglo XII), así como el pensamiento teológico y las propuestas combinatorias de Ramón Llull (1232-1316). Para justificar estas conclusiones la obra se compone de una introducción y seis capítulos que reúnen diversas publicaciones académicas dispersas sobre el tema.

La Introducción recoge el fuerte impacto de la cábala provenzal a partir de 1220 en Gerona a través de Isaac el Ciego y su seguidor Najmánides o Saporta, en versión cristiana. A partir de 1250 la cábala de Gerona quedaría como única depositaria de la cábala provenzal. Frente a la visión uniforme de la cábala gerundense, Idel reconstruye las más importantes polémicas que se entablaron entre sus diversos representantes, ya sea sobre los diez atributos o manifestaciones divinas o sobre si se deberían reducir a dos o sólo una; ya sea sobre si los atributos divinos constituyan algo exclusivamente interno o se trata más bien de manifestaciones externas; ya sea sobre si el predomino en el sistema astral se debe asignar al sol o a la luna; ya sea acerca del procedimiento esotérico de la trasmisión del saber; ya sea por su mayor o menor interés en su proyección pública en Barcelona o incluso fuera de la corona de Aragón, en Toledo y otras ciudades de Castilla; ya se centraran en el Comentario al Pentateuco o en la obra talmúdica Séfer Yetsirá (Libro de la creación) - con un fuerte impacto en la cábala de Barcelona a partir de 1270 - o según manifestaran una mayor o menor apertura a otras tradiciones de pensamiento, especialmente después de la llegada a Barcelona de Abufalia, con la introducción de La guía para perplejos de Maimónides, o de la tradición asquenazi procedente de centro Europa. Posteriormente se analiza el fuerte impacto de la teoría de los diez atributos divinos de la cábala, reducidos a sólo tres - la misericordia, el juicio o discernimiento y el temor -, en la noción de conjunción combinatoria total o mezcla de contrarios, así como en el concepto trinitario de divinidad de Ramón Llull.

1) La historia de la cábala en Barcelona. Se sitúa a partir de 1250 con el protagonismo principal de Najmánides, aunque ahora se postula la presencia de un núcleo anterior incluso al de Gerona. Se prolongan así algunas propuestas de Gershom Scholem sobre la cábala en España con anterioridad a 1250, aunque según Ideal las controversias gerundenses habrían tenido desde su inicio su correspondiente réplica en Barcelona, donde hay constancia de la presencia de la cábala desde al menos 1175 y donde se habría formado Najmánides. Se conciben ahora como dos escuelas diferenciadas con orígenes propios, aunque la partida de Najmánides a Israel en 1267 hiciera que el centro gerundanse prácticamente desapareciera. Frente al concepto esotérico de la cabala, sus seguidores un tanto heterodoxos de la cábala de Barcelona, especialmente Ben Andret en sus Comentarios sobre la liturgia de los días de fiesta, entendieron que sus doctrinas deberían difundirse sin respetar el hermetismo bibliocéntrico de su maestro respecto de los secretos que guardaba el Comentario al Pentateuco, aunque en el fondo no hubiera tantas diferencias entre ellos. Finalmente, en 1270 llegaría a Barcelona Abraham Abulafia, después de su marcha en 1260 a Palestina e Italia, y de haber conocido La guía de perplejos de Maimónides. Sin embargo pronto abandonaría la filosofía para dedicarse exclusivamente a la cábala a partir del estudio del Séfer Yetsirá (El libro de la creación). Se dedicaría en exclusiva a la cábala extática o mística judía, interesada especialmente por las combinaciones de letras y de atributos. De este modo los cambios innovadores introducidos por el intérprete pueden desempeñar un papel fundamental en la interpretación de un texto, sin guiarse por los criterios estrictamente conservadores a ultranza de los seguidores de Najmánedes. De todos modos esta 
corriente de la cábala gerundense prefirió el uso del lenguaje filosófico y prácticamente desapareció a mitad del siglo XIV, especialmente de por el escepticismo que la propia comunidad judía mostró ante la cábala esotérica de Najmánides.

2) Najmánides: la cábala, Halajá y liderazgo espiritual. Se revisan las tesis de I. Tishby, seguidor a su vez de las tesis de Gershom Scholem. Le atribuyen a Najmánides una posición subordinada a Sagi Nehor en la cábala de Gerona, aunque en ambos casos había ejercido un fuerte influjo la lectura del Libro sobre la claridad recibido a través de la cábala provenzal. Sin embargo ahora se le atribuye a Najmánides una posición cabalística diferenciada respecto del resto de los miembros de su comunidad, aunque en todos los casos se aceptara la mística y el mandado de guardar los secretos de la cábala. Sin embargo Najmánides había defendido la Halajá, donde se distancia de la tesis de los diez atributos asignado a la divinidad, así como de la difusión de la filosofía de Maimónides. De ahí que Najmánides defienda la existencia de ciertos secretos que sólo deben ser conocidos por vía oral por unos pocos. Se trata de secretos que constituyen el "camino de la verdad" que pone en correlación el mundo inferior y el superior, como ahora sucede en el Comentario al relato de la creación. O con la correspondencia que se establece entre los siete días de la creación y los atributos divinos. Sin embargo Najmánides rechaza servirse del procedimiento hermenéutico del cálculo de letras (guematría) o de la especulación filosófica neoplatónica o aristotélica. En su opinión, la cábala se debe justificar exclusivamente en nombre de la exégesis bíblica, fomentando la custodia de sus secretos, o la ahora denominada halajá, como a su modo de ver corresponde al ejercicio de cualquier tipo de liderazgo espiritual En cualquier caso Sem Tov en 1325 o de Cordovero en el siglo XVI consideran que se trata de dos escuelas cabalísticas diferenciadas, a pesar de pertenecer a la misma comunidad.

3) Dignitates y Kavod: dos conceptos teológicos en el misticismo catalán. Se comparan las propuestas de Ramón Llull respecto de las dignidades o atributos divinos y el Kavod o la denominación que reciben en el misticismo judío, así como sus consecuencias en el modo de concebir el arte combinatorio luliano, como ya habría sido señalado por Pico de la Mirándola. Por su parte ahora también se analizan las propuestas de Millás-Vallicrosa, aunque se señalan las diferencias entre la Causa Causarum del neoplatonismo cristiano y la Corona como primer atributo divino en el misticismo judío. Najmánides conocía bien el cristianismo y tenía buenas relaciones con el rey de Aragón, auque su estricto esoterismo le impedía mantener una relación de auténtico diálogo en cualquier forma de controversia.

4) Abraham Abufalia. Un cabalista místico. Nacido en 1240 en Zaragoza, se crió en Tudela, marchó en 1260 a Palestina, pasó por Italia donde conocería La Guía de Perplejos de Maimónides a través del filósofo judío Hilel de Verona, para en 1270 volver a Barcelona, En 1279 volvería de nuevo a Italia, en 1280 trató sin éxito de entrevistarse con el Papa, y para ir a continuación a Sicilia donde moriría en 1279. Su misticismo askenasí de procedencia centroeuropea era más abierto a la filosofía y a la difusión del pensamiento. Defendería una mística extática en la que separa la actividad profética y la contemplación de los atributos divinos, mediante la simple combinación de las letras de los nombres divinos, que le permitirían conocer la estructura interna del mundo divino. De este modo el alma del místico se podría elevar hasta la carroza celeste mediante la repetición de palabras y de himnos litúrgicos, al modo cultivado por el judaísmo askenazí procedente del valle de Rin. Se consigue así el estado extático de unión mística al modo señalado por la cábala, adquiriendo una conciencia del yo profético-mesiánico mediante el recurso a numerosos procedimientos matemáticos de descomposición más que de composición, como solía ser habitual. Surgen así los maestros itinerantes que divulgan la cábala allí por donde pasaban, adoptando una actitud exotérica muy 
distinta a la que había caracterizado a la cábala en sus inicios. De hecho la influencia de Abufalia sería muy grande por todo el imperio de Bizancio, especialmente Italia.

5) Ramón Llull y la cábala extática. Se compara el recurso de Llull a un arte combinatorio universal o enciclopédico con la cábala extática o profética, al modo propuesto por Abufalia, al menos según Pico de la Mirándola. Sin embargo el primero fomentó la combinación de todo tipo de letras (revolutio alphabetorum) para lograr así ampliar al máximo el saber racional, mientras que el segundo sólo trató de combinar entre sí los atributos o nombres divinos para alcanzar un conocimiento místico extático o profético de los propios designios divinos, como al menos sucede en Abraham Abulafia. Habría sido en 1270 cuando Abulafia en Barcelona había recibido una revelación especial mediante la que se debería generalizar para todos los seres el arte combinatoria aplicado hasta entonces sólo a los nombres divinos. Al menos así también lo propondrá posteriormente Ramón Llull en la Logica nuova (1305) en dependencia a las propuestas previa de la cábala de Abulafia. Se resaltan así la importancia de las técnicas estáticas esquenazis en el origen del arte combinatorio, ya sea de Abulafia o de Ramón Llull, como ahora se justifica analizando las semejanzas existente entre los diagramas usados en cada caso.

6) Esoterismo esquenazi y cábala en Barcelona. Se describe la cábala teosófica-teúrgica desarrollada en Barcelona entre 1270 y 1330, de un modo un tanto centrípeto o nomiano que otorgaba una centralidad a los mandamientos y al cumplimiento de la Torá como primera obligación religiosa, a diferencia de lo que sucede habitualmente en otras corrientes del judaísmo. $\mathrm{Su}$ figura central sería Abraham Abulafia, junto a otros cabalistas, en dependencia con la tradición esquenazi proveniente del sur de Alemania, concretamente de Ashkenas, de Italia o sin más del Oriente. Se comprueba así la penetración de la mística estática y de la liturgia esquenazí en el Seferad del siglo XIII, con la consiguiente polémica sobre el significado teológico de las respectivas letras del alfabeto hebreo. Además, así lo certifica la visita a Barcelona del Rabí Axelrod de Colonia en 1260 o el testimonio de Sen Tov de Carrión de los Condes en 1325. En cualquier caso se describe un ambiente multicultural muy enriquecedor donde rivalizaban las ciudades de Barcelona, Toledo y Colonia en el dominio cabalístico de las ciencias ocultas.

Para finalizar una conclusión crítica. Sin duda la presencia de la corriente esquenazí y safardí en la tradición de la cultura hebrea española se remonta al siglo XIII. Por su parte, resulta fácil de pensar que su influencia pudo ser mutua, pudiéndose concebir como un factor decisivo del impacto del pensamiento de Maimónides, Averroes o del propio Ramón Llull en Centroeuropa. Por su parte también es de señalar la importancia otorgada a determinados diagramas alfabéticos cabalísticos, adelantándose al uso frecuente que la lógica barroca moderna hará de esta herramienta heurística. En cualquier caso la monografía reconstruye la creciente apertura de la cábala judía a un conjunto de múltiples tradiciones que, como ocurre con La guía de perplejos de Maimónides, acabarían ejerciendo una fuerte influencia en los Proverbios morales de Sem Tob de Carrión (1290-1369).

CARLOS ORTIZ DE LANDÁZURI Universidad de Navarra

\section{CORTI, ENRIQUE. Oír, entender, argumentar: Lectura de Proslogion y De Grammatico de Anselmo de Canterbury, Buenos Aires: Miño y Dávila, Colección Lejos y cerca, 2016, 304 pp.}

El propósito de este libro queda claramente expresado por el Autor en el Prefacio (pp. 9-10), donde nos adelanta su intención de abordar cuestiones centrales que Anselmo trató en sus dos primeras obras: Monologion (1076) y Proslogion (1077-78), cuyos títulos iniciales fueron "exemplum meditandi de ratione fidei" y "fides quaerens intellectum" respectivamente. Corti deja también debidamente aclarado 
que, si bien su interés estará centrado en el célebre unum argumentum desarrollado en Proslogion, no podrá evitar la referencia a Monologion es insoslayable en orden a la comprensión cabal de la evolución del pensamiento de Anselmo, dado el vínculo que él mismo describe entre ambos textos en el Prooemium a Proslogion. Efectivamente, in ipso cogitationum conflictu se le presenta el que será su famoso argumento, y lo hace de modo tal que le resulta inapelable. De ahí que aparezca como necesario y conveniente presentar "la génesis que conduce desde los argumentos múltiples encadenados en Monologion al argumento único de Proslogion". Este último, que marcaría el rumbo que Anselmo mantendrá en obras posteriores, ha merecido innumerables exégesis, la mayoría de las cuales, según sostiene Corti, se centra en los primeros capítulos de la obra omitiendo el contexto amplio en el que ha de ser entendido. Es por ello que el Autor se propone examinar la génesis del argumento único que ha de rastrearse en Monologion, pero, además, colocar el texto de Proslogion en su conjunto en el marco de la consideración del lenguaje, la significación y las categorías lingüísticas que, en opinión del medievalista argentino, ha sido esencial al rumbo que Anselmo imprimiera a su pensamiento. Por la misma razón, Corti entiende que debe incluir en este mismo libro la lectura del tratado De grammatico (ca. 1080) en el que Anselmo "despliega un análisis minucioso del lenguaje en su uso común coloquial y en su uso no común o propio a efecto de establecer una distinción de la mayor relevancia para comprender su pensamiento, la argumentación, y los vínculos entre gramática y lógica: la existente entre significatio y apellatio”.

En el libro se despliegan así dos medulosos estudios de Corti que preceden, respectivamente, a la lograda traducción española (con el texto latino a página enfrentada) de Proslogion y De Grammatico (en el caso de este último texto, realizada por Antonio Tursi).

Oír, entender, argumentar es, pues, el fruto de un trabajo exhaustivo y minucioso de Enrique Corti, una obra rica en argumentaciones y análisis de gran penetración, en donde se desarrollan de manera erudita, por una vía que no ha sido regularmente la más transitada, tópicos capitales del pensamiento anselmiano. Es ése el camino elegido por este experimentado investigador y profesor de Filosofía Medieval, cuya experticia en el tema queda plasmada de manera íntegra y acabada en esta obra que nos ofrece la colección "Lejos y Cerca" dirigida por Silvia Magnavacca.

MICAELA MARRONE UNR

Facultad de Humanidades y Artes de Rosario, Santa Fe, Argentina

\section{ABŪ NAṢR AL-FĀRĀBĪ, Las filosofías de Platón y Aristóteles, traducción, introducción y notas de Rafael Ramón Guerrero, Ápeiron Ediciones, Madrid, 2017. 172 páginas.}

Rafael Ramón Guerrero, titular de la cátedra de Filosofía Medieval de la Universidad Complutense de Madrid y uno de los más prestigiosos investigadores en este área, suma a su larga lista de obras -entre las que destacan El camino de la felicidad ${ }^{l}$, Obras filosófico-políticas ${ }^{2}$, La recepción árabe del De anima de Aristóteles: Al-Kindi y Al-Fārābi ${ }^{3}$, El conocimiento o ciencia profética en Al-Kindi y Al-Fārābi ${ }^{4}, E l$ compromiso político de Al-Fārābi ${ }^{5}$, Al-Fārābī lógico: su "Epístola a la introducción al arte de la

1 Al-Farabi, El camino de la felicidad (Kitab al-tanbih 'alà sabil al-sa'ada), traducción, introducción y notas Rafael Ramón Guerrero, Ed. Trotta, Madrid, 2002.

2 Al-Farabi, Obras filosófico-políticas, edición Rafael Ramón Guerrero, CSIC, Madrid, 1992.

3 Ramón Guerrero, Rafael, La recepción árabe del De anima de Aristóteles: al-Kindi y al-Farabi, CSIC, Madrid, 1992.

4 Ramón Guerrero, R., "El conocimiento o ciencia profética en al-Kindi y al-Farabi", Actas de las Jornadas de Cultura Árabe e Islámica (1978), Madrid 1981. pp. 353-358.

5 Ramón Guerrero, R., "El compromiso político de Al-Farabi: ¿Fue un filósofo si'i?”, Actas de las II Jornadas de Cultura Árabe e Islámica (1980), Madrid 1985. pp. 463-478. 
lógica "6, La Ética a Nicómaco en la obra de Al-Fārāā̄ $\bar{\imath}^{7}$ Al-Fārābī: el concepto de ser ${ }^{8}$, Razón práctica e Intelecto agente en Al-Fārāẩ $\vec{\imath}^{9}$, Al-Fārābĩ: Epistola sobre los sentidos del término intelecto ${ }^{10}$ - una nueva traducción, imprescindible para la comprensión del pensamiento filosófico islámico y su relación con el legado griego.

El presente volumen recoge dos obras completas de Al-Fārābī y dos fragmentos de una tercera. La primera de ellas, que lleva por título La filosofía de Platón ha sido traducida por Ramón Guerrero a partir de la edición de A. Badawi, teniendo en cuenta también la edición de F. Rosenthal y R Walzer. La segunda obra, La filosofia de Aristóteles, lo ha sido a partir de la edición de M. Mahdi. Finalmente a ellas se une un apéndice en el que se ofrece la traducción del Prólogo y el Tratado Primero del Sumario de las Leyes de Platón basada en la edición de T. A Druart.

Estas tres traducciones están encabezadas por una amplia introducción que Ramón Guerrero estructura en tres partes dedicadas, cada una de ellas, a contextualizar y analizar un aspecto central del contenido temático de las mismas. La primera parte de la introducción está dedicada a la presencia del pensamiento de Platón y Aristóteles en el mundo islámico. A lo largo de sus páginas, Ramón Guerrero destaca el carácter religioso y sagrado que el Islam ofrece al conocimiento, no sólo sobre Dios sino también sobre el universo, la naturaleza y el hombre, hecho que da explicación a la fácil asimilación de la filosofía en este marco cultural. Se presenta, además, el proceso de traducción del pensamiento filosófico greco-romano, realizado fundamentalmente en Bagdad y su empleo inicial para los procedimientos dialécticos derivados de las disputas teológicas con los cristianos.

Ramón Guerrero apoya en este punto la interpretación de D. Gutas que entiende el proceso de transmisión como un fenómeno social promovido por la sociedad abasí en su conjunto, fruto de un esfuerzo metodológico consciente y deliberado. Asimismo se destaca el conocimiento por parte de los árabes de casi todos los diálogos de Platón de los cuales, sin embargo, sólo se conservan en árabe resúmenes o exposiciones de sus escritos. En cambio, de Aristóteles, los árabes conocieron prácticamente toda su obra, venerada hasta tal punto que el filósofo griego fue conocido como el Maestro primero, reconocido por Averroes como la culminación de toda la filosofía anterior.

La segunda parte de la introducción está dedicada a analizar en detalle el manejo que Al-Fārābī hizo de la obra de Platón y el influjo que el platonismo, sobre todo en su vertiente política, tuvo sobre su pensamiento. Ramón Guerrero analiza en este apartado la polémica iniciada por la interpretación de Leo Strauss que distingue, en la producción de Al-Fārābī, entre obras esotéricas y exotéricas basándose en la idea de la necesidad que este pensador habría tenido de ocultar algunos contenidos filosóficos polémicos de la mirada de la teología musulmana. Frente a la aparente incoherencia de las tesis sostenidas por Al-Fārābī en estos supuestos dos tipos de escritos, Ramón Guerrero se distancia de la visión de Strauss mostrando el perfecto encaje que hay ente las ideas de sus obras, situando cada una de sus posturas en el marco filosófico y religioso de su época. A continuación, se presenta un análisis resumido del contenido de la primera obra, La filosofia de Platón, en la que Al-Fārābī ofrece un estudio sobre la naturaleza del hombre y del

6 Ramón Guerrero, R. "Al-Farabi lógico: su "Epístola de introducción al arte de la lógica”, Homenaje al profesor Dario Cabanelas Rodriguez, O.F.M., con motivo de su LXX aniversario, Vol.1, Granada 1987. pp.4568.

7 Ramón Guerrero, R., "La Ética a Nicómaco en la obra de Al-Farabi”, Ética y sociología: estudios en memoria del profesor José Todoli, O.P. coord. por Luis Méndez Francisco, Salamanca 2000. pp. 201-212.

8 Ramón Guerrero, Rafael, "Al-Fārābī: el concepto de ser", Revista de Filosofía, no 11, 1994. pp. 27-50

9 Ramón Guerrero, Rafael, "Razón práctica e Intelecto agente en Al-Fārābī”, Tópicos: Revista de Filosofia, $\mathrm{n}^{\mathrm{o}} 18,2000$. pp. 73-95

10 Ramón Guerrero, Rafael, "Al-Fārābī: Epístola sobre los sentidos del término intelecto", Revista española de filosofia medieval, $\mathrm{n}^{\circ}$ 9, 2002, pp. 215-224 
mundo, un análisis epistemológico de cada ciencia y una exposición de contenidos de los diálogos platónicos concluyendo con una referencia a las Cartas de Platón.

La tercera parte de la introducción está consagrada al estudio del influjo de Aristóteles sobre el pensamiento de Al-Fārābī. Tal como señala Ramón Guerrero, el filósofo árabe pudo leer gran parte de la obra de Aristóteles y su conocimiento directo de ella está fuera de toda duda. No obstante, la lectura que Al-Fārābī hace de Aristóteles está profundamente matizada por la interpretación neoplatónica. Signo de ello es, por ejemplo, su pertenencia a la tradición que intenta conciliar los pensamientos de Aristóteles y Platón, iniciada por Antíoco de Ascalón. No obstante, existiendo pruebas claras de que nuestro filósofo conocía tanto al Aristóteles neoplatónico como su versión inalterada, llama la atención la insistencia del filósofo musulmán en defender premisas pertenecientes a la primera tradición. Para explicar su posición, Ramón Guerrero rastrea el contenido de la obra Concordia entre el divino Platón y Aristóteles ofreciendo pruebas evidentes de que Al-Fārābī distinguía claramente entre el objetivo y el tema de la Metafísica y el de la obra pseudo-aristotélica Teología. Pero si ello es así y Al-Fārābī comprendía perfectamente en qué medida la tradición alteraba la obra aristotélica, la razón para mantener una lectura de inspiración platónica de los textos aristotélicos no sería, por tanto, una confusión sino un hecho derivado de la convicción por parte de Al-Fārābī de que la felicidad sólo puede alcanzarse en comunidad. De ahí, según Ramón Guerrero, que su filosofía se inspira y sustenta en el ideal de la República que viene a configurarse como meta última de todos los esfuerzos filosóficos.

Esta introducción es continuada inmediatamente por las traducciones de las tres citadas obras. El volumen se cierra con una nutrida y actualizada bibliografía centrada no sólo en la obra de Al-Fārābī sino también en los estudios dedicados a la asimilación de Platón y Aristóteles en el mundo islámico.

ANA MARIA C. MINECAN

Universitatea Babes-Bolyai, Cluj

\section{PIETRO DI GIOVANNI OLIVI FRATE MINORE: atti del XLIII Convegno internazionale, Assisi, 16-18 ottobre 2015, Spoleto, CISAM, 2016, 518 pp.}

Se han publicado las actas del XLIII Convegno internazionale della società di studi francescani, dedicado en este año a Pietro di Giovanni Olivi frate minore. El resultado es una monografía con notables puntos de interés, que viene a complementar otros estudios colectivos publicados en los últimos años sobre el pensamiento del minorita provenzal, particularmente C. König-Pralong, O. Ribordy, T. SuarezNani (eds.), Pierre de Jean Olivi. Philosophe et théologien, Berlin, De Gruyter, 2010.

Se ha mantenido la estructura del encuentro para la publicación de las Actas, si bien no se han transcrito los debates posteriores. De esta forma, siguiendo el orden, la primera intervención correspondía a Jacques Chiffoleau y a Clément Lenoble (EHESS, CIFIAM-UMR, Lyon), quienes en su "Les Frères mineurs dans les sociétés de Provence et du Languedoc au temps d'Olivi" trazaron un marco amplio y detallado para comprender mejor los intereses minoríticos en la segunda mitad del siglo XIII. Se trata del punto de arranque de la obra, que permite encuadrar las ponencias posteriores.

La profesora Tiziana Suarez-Nani (Université de Fribourg Suisse), en "Il profilo intellettuale di Olivi e il progetto di una 'filosofia spirituale"' defiende que el proyecto filosófico oliviano puede ser considerado una verdadera filosofía espiritual, que ha madurado el proyecto aristotélico, proponiendo una postura alternativa a partir del concepto de libertad y bajo el axioma de la superioridad del espíritu. Por esa razón, Olivi es un pensador irreductible a los esquemas de su tiempo. Lo expresa en una buena síntesis: "aristotelico senza aderire all'empirismo di Aristotele, platonico senza adottare l'esemplarismo delle idee, bonaventuriano senza condividere la reductio artium ad theologiam, Olivi è stato 'soltanto' e sopratutto Olivi..." (pp. 129-130). 
Fortunato Iozzelli (Pontificia Università Antonianum, Roma), se concentra en "Aspetti dell'esegesi biblica di Pietro di Giovanni Olivi”, mostrando sus coincidencias con la exégesis bíblica del siglo XIII y destaca la lectura que hizo de la parábola del hijo pródigo. Por su parte, Antonio Montefusco (HeinrichHeine Università, Düsseldorf), señala las palabras de léxico vulgar en los manuscritos de Olivi y sugiere la existencia de "il progetto bilingue di Olivi e la memoria dissidente" para escribir en su lengua provenzal.

Damien Ruiz (UMR TELEMME, Aix-Marseille Université), retoma el tema del comentario oliviano a la regla franciscana en "La Règle et l'Ordre chez Pierre de Jean-Olivi" y entiende que la postura del minorita de Béziers suponía una sublimación de la regla de la orden. Es una lástima que no se haya publicado en las Actas la intervención encargada a Andrea Tabarroni (Università di Udine), titulada "Le idee politiche di Olivi nel quadro della tradizione minoritica". Sí que se ha publicado la ponencia de Giovanni Ceccarelli (Università di Parma), sobre "Il Tractatus de contractibus di Olivi nel discorso economico dei Frati minori", en la que se muestran las posibles contradicciones del discurso oliviano al estudiar los mecanismos del capitalismo mercantil y al defender un mercado orientado hacia los bienes de la colectividad.

Marco Bartoli (Università LUMSA, Roma), en "Pietro di Giovanni Olivi: una ecclesiologia minoritica?" concluye que, pese a que no exista un tratado de eclesiología oliviana, la piedra de toque de la misma se tendría que hallar en la famosa quaestio sobre la indulgencia de la Porziuncola, según la cual, la pobreza material de la Iglesia sería el sostén de su autoridad espiritual.

Paolo Vian (Biblioteca Apostolica Vaticana), distingue en "L'interpretazione della storia nella Lectura super Apocalipsim di Pietro di Giovanni Olivi e i contesti della sua ricezione" el valor intrínseco del comentario, calificado como uno de los más importantes de la exegética medieval, y su recepción, que compara al grano de trigo de los Evangelios, por su fecundidad.

La última parte del libro, incluyendo en parte la ponencia de Vian, se orienta más hacia la recepción de las ideas de Olivi. Así, Louisa A. Burnham (Middlebury, University of Vermont), en "The Angel With the Book" se centra en el culto a Olivi, especialmente en el caso de Prous Boneta de Montpellier y trata el problema del ángel de la revelación en Olivi, y analiza la lectura que hicieron los beguinos, con el fin de subrayar el contexto apocalíptico de la recepción de los textos olivianos.

Alberto Forni retoma el tema de la influencia de Olivi en Italia en su ponencia "Pietro di Giovanni Olivi nella penisola italiana: immagine e influssi tra letteratura e storia”, centrándose especialmente en el debatido tema de su relación con Dante, y Paolo Evangelisti (Archivio storico Camera dei Deputati), en su "Da Guillem Rubió a Joan Bassols. L'eredità di Olivi nei territori iberici" subraya los ecos de Olivi en el pensamiento de la Corona de Aragón. Al final del texto de Evangelisti se encuentran unos gráficos que valdría la pena que se desarrollasen en próximos trabajos. En ellos se subrayan obras con referencias textuales derivadas de las obras teológico-exegéticas, sobre la pobreza o la perfección evangélica o sobre el Tratado de los contratos de Olivi. Algunos de estos autores han sido más estudiados (Arnau de Vilanova, Eiximenis, Guiral Ot), mientras que otros como Ponç Carbonell, Guillem Rubió, Antoni Andreu, Joan Bassols... merecen mayor atención por parte de los estudiosos.

Las palabras conclusivas de Roberto Lambertini (Università di Macerata) ponen punto y final a estas jornadas de gran interés para la medievalística y que sirven para profundizar en el conocimiento de Olivi, uno de los grandes nombres del pensamiento del siglo XIII, cada día más recuperado del injusto olvido en el que ha permanecido durante centurias.

RAFAEL RAMIS BARCELO

Universitat de les Illes Balears - IEHM 


\section{CLAUS A. ANDERSEN, Metaphysik im Barockscotismus. Untersuchungen zum Metaphysikwerk des Bartholomaeus Mastrius. Mit Dokumentation der Metaphysik in der scotistischen Tradition ca. 1620-1750, John Benjamins Publishing Company, Amsterdam, 2016, 993 pp.}

Hay obras que pueden calificarse de excepcionales, porque representan una contribución fuera de lo común en el panorama filosófico. Es el caso de este libro del Dr. Claus A. Andersen, de la Universidad de Mannheim, fruto de su elaboradísima tesis doctoral, defendida en 2014, que bien merecería la categoría de tesis de habilitación por la profundidad y amplitud de la temática abordada, y por el hecho de colmar una laguna historiográfica que sólo había sido analizada parcialmente hasta ahora.

Trabajos sobresalientes de Marco Folivesi, Sven K. Knebel, Jacob Schmutz o Daniel Herder, que complementan otros igualmente fundamentales, aunque más antiguos de Antonino Poppi, Ludger Honnefelder... habían sido piezas angulares para estudiar la transición desde el escotismo tardomedieval al barroco, y la discusión de los escotistas con la escuela jesuítica del siglo XVII. A esta honrosa nómina debemos sumar ya el voluminoso y elaboradísimo libro de Claus A. Andersen.

Esta obra estudia la metafísica en el escotismo barroco, con una atención especial a la obra del franciscano conventual Bartolomeo Mastri (1602-1673), natural de Meldola (Italia), y conocido como el "príncipe de los escotistas" y que escribió obras individuales y también a cuatro manos con su correligionario Bonaventura Belluto (1600-1676). El libro recoge abundante documentación de la recepción de las ideas de Escoto en el período que abarca desde 1620 hasta 1750. Sin duda, se trata de una contribución que sobrepasa el simple análisis filosófico de esta corriente: es un auténtico diálogo con toda la filosofía barroca y con el escotismo precedente y posterior.

La obra está dividida en una introducción y cinco partes, que concluyen con un rico apéndice de autores tratados, así como con una completísima bibliografía. Como salta a la vista, no podemos detenernos en cada uno de los apartados de esta obra de casi mil páginas. Para que el lector se haga una idea más certera de su contenido, expondremos brevemente el contenido y temática de cada una de las partes y capítulos, y luego pasaremos a un análisis de ciertas cuestiones que consideramos fundamentales, sobre todo desde un prisma historiográfico.

Los dos primeros capítulos son introductorios, aunque son los fundamentos de la obra y una discusión muy elaborada con la historiografía. Hallamos la definición y características del Barockscotismus y una explicación de por qué, según el autor, estamos ante un reto historiográfico, atendiendo a la recepción interna y externa de Escoto en la primera Modernidad.

La primera parte trata del saber metafísico y comprende los capítulos tercero a quinto. El capítulo 3 estudia el concepto de la metafísica y el problema de su fundamentación en la obra de Mastri, mientras que el siguiente trata del problema de la metafísica in se y de la metafísica in nobis en la tradición escotista desde Trombetta hasta Mastri. El capítulo 5 está destinado a profundizar en la pregunta por el método, detallando el orden doctrinal (ordo doctrinae) y la presentación de la metafísica en la obra de Mastri.

La segunda parte versa sobre el concepto de ser. El capítulo 6 analiza los seres como palabra y concepto, detallando la estructura y el análisis de los conceptos del ser en la escolástica barroca, explicitando los problemas de la deriva nominalista y una serie de distinciones fundamentales sobre el concepto del ser y del ente. El capítulo séptimo se ocupa de la transcendencia (genus transcendentale) e indiferencia del concepto de ser, mientras que el capítulo octavo se dedica a precisar el concepto de ser. Especialmente relevante es el capítulo 9, dedicado al concepto análogo-unívoco del ser, que parte desde Suárez y llega a Mastri, pasando por otros autores, concretando la opinión de los mismos (análogo-equívoco, equívoco puro, unívoco-análogo), hasta llegar a la crítica de Mastri de la metafísica suareciana.

Igualmente, para la historia de la filosofía resulta fundamental la tercera parte, sobre los "modos" del ser, que se inicia con el capítulo 10, central en el el hilo conductor del libro, sobre los modos intrínsecos y extrínsecos de ser, en el que se dirime el debate de fondo entre la tradición escotista y la tradición 
jesuítica. En el capítulo 11 se estudia la existencia, lo finito y lo infinito como modos de existencia en las criaturas y en Dios.

La cuarta parte se titula "Principia, Passiones, Divisiones". El capítulo 12 está dedicado a la discusión que hizo Mastri de esta tríada de conceptos, mientras que el decimotercero estudia los requisitos de los trascendentales, explicitando la divergencia entre Mastri, Suárez y Pasqualigo, concluyendo con las passiones entis positivae y el problema de la adición, confrontando a Mastri y a Suárez. El capítulo decimocuarto está dedicado a los trascendentales simples: unum, verum y bonum, mientras que el capítulo 15 trata el tema de los trascendentales disyuntivos en la tradición escotista (primero desde Antoni Andreu hasta Suárez) y luego hasta Mastri.

La quinta parte - una de las más importantes de la obra- está dedicada a la teoría de la distinción de Mastri. El capítulo 16 estudia la ubicación de la teoría de las distinciones en el marco del cursus philosophicus, y el siguiente analiza la reorganización que hizo el franciscano italiano de la teoría tradicional de la distinción, y concluye con las críticas que recibió el propio Mastri en la reducción de la distinción modal. El capítulo 18 analiza la distinción ex natura rei en la distinción real y formal, a través de un estudio genealógico de la doctrina de la distinción, mientras que en el siguiente se comenta la distinción de razón en la interpretación escotista.

Las conclusiones de la obra se distribuyen en cuatro puntos: 1) la orientación básica de concepto metafísico en el ser real en general en el pensamiento escotista barroco; 2) en particular, el análisis de las principales concepciones metafísicas de Mastri; 3 ) un estudio de las principales fuentes del escotismo moderno y 4) y las consecuencias historiográficas de todo lo anterior. Claus A. Andersen destaca, ante todo, el papel fundamental de Mastri en las reelaboración de la metafísica escotista, en diálogo crítico con la tradición jesuítica y la influencia que tuvo en el escotismo posterior.

Andersen valora especialmente a Zacaria Pasqualigo y Raffaele Aversa, dos tratadistas de la generación posterior a Suárez, con los que Mastri dialoga. Muestra las continuidades y las continuidades de la filosofía ad mentem Scoti, al tiempo que subraya las transformaciones internas y externas de la tradición escotista, especialmente con el contacto con la tradición jesuítica. Se trata, en definitiva, de un momento destacado para el desarrollo conceptual de la metafísica europea, que nunca se había puesto de manifiesto de forma suficiente.

El apéndice incluye importante información sobre la metafísica escotista entre los años 1620-1750 y una breve descripción de los respectivos enfoques metafísicos de autores como Meurisse, Rapinaeus, Poncius, Lallemandet, Dupasquier, Frassen... hasta llegar a Gomez Mexia y Ubillos.

Sentado lo anterior, podemos afirmar que gracias a la investigación de Claus A. Andersen se ha podido avanzar mucho en un tema abierto en los últimos años especialmente por Marco Forlivesi, en el que se hacen notables precisiones conceptuales, que no podemos tratar exhaustivamente, aunque sí podemos destacar algunas de las más interesantes, a nuestro parecer.

En primer lugar, la periodificación del escotismo barroco en cuatro fases: la incial (1600-1640), con nuevos tratados en el seno de la tradición formalista, que dieron lugar a las innovaciones de Rapinaeus, Meurisse y otros, influidos por Suárez, 2) la fase central (1640-1650), en la que brillaron Mastri y Belluto, aunque también Poncius y Lallemandet; los primeros fueron críticos de la metafísica jesuítica, mientras que, por ejemplo, Lallemandet fue más ecléctico; 3) la fase de los epígonos y de los críticos, que duró de 1650 a 1750, en la cual hallamos el grueso de los escotistas influidos por Mastri, que en ocasiones se alejaron de él, mientras que en otros casos concordaron con el estudioso italiano; 4) la fase final establecida hacia 1750, con figuras menores que, sin embargo, ejercieron una cierta influencia en la filosofía del siglo XVIII.

La segunda consecuencia, en esta ocasión de carácter historiográfico, es advertir que los franciscanos, sobre la base escotista, elaboraron tal vez los comentarios más sutiles a la metafísica de Aristóteles de la 
época moderna. Suárez fue, si se nos permite la expresión, un "escototomista", que llenó los odres tomistas de escotismo, en una obra de la cual es deudora toda la filosofía moderna, desde Descartes a Kant. Esa síntesis fue también muy importante para la propia evolución del escotismo, así como para la elaboración de lo que se llamó el "suarismo". Cuando Andersen habla de la recepción interna y externa de Escoto en la época moderna apunta a una serie de problemas historiográficos y conceptuales muy destacados, sobre los que cabría volver en futuros trabajos.

Uno de ellos sería cómo los franciscanos pudieron luchar contra la recepción suarista de Escoto y volver a una interpretación más cercana a lo que había sido la "tradición escotista". Y es que la obra de Mastri (y de Belluto), como bien se muestra en el libro, se escribió en continuo diálogo con (y muchas veces en contra de) las doctrinas de Suárez y de algunos teóricos posteriores. Véase el énfasis de Andersen al subrayar, por ejemplo, las diferencias entre Sirectus, Suárez y Mastri en el tratamiento de los "trascendentales disyuntivos".

Una tercera consecuencia es la riqueza de Mastri en el seno de la propia tradición escotista, al incorporar el acervo anterior, adoptando y criticando algunas innovaciones del suarismo. Por ejemplo, en el cuadro 8 , de la p. 705, se detalla la teoría de los modos de distinción de tomistas, escotistas y otros en la lectura de Mastri. Especialmente destacada es la distinción entre los siete modos de distinción de los Scotistae veteres (Pere Tomàs y otros) y los Scotistae recentiores (de primera generación del escotismo barroco), que distinguían entre real (esencial, subjetiva, objetiva), formal (ex natura rei y formal) y racional, hasta llegar a la de Mastri, que distinguía los primeros géneros (rationis y ex natura rei) con subdivisiones que matizaban los modos "tradicionales" de la distinción escotista.

No podemos seguir examinando diferentes puntos de interés de este extenso libro, de lectura densísima, aunque muy bien estructurado. La precisión conceptual del Dr. Andersen, ayudada por un exquisito tratamiento filológico y un análisis concienzudo de las fuentes permiten decir que estamos ante una obra extraordinaria, de las que marcan época y a la cual no podemos formular ninguna objeción global. Al contrario, el proceder y el resultado nos parecen excelentes.

Sólo en la tesitura de formular algunos comentarios, nos atrevemos a indicar algunos puntos que tal vez el autor podría abordar en el futuro, pues el libro deja con ganas de conocer algunas prolongaciones o vínculos que en la obra sólo quedan insinuados. Diremos solamente cuatro de ellos.

El primero sería un análisis en paralelo de la forja de la tradición del "escotismo barroco" y del "suarismo". En la obra hay abundantes alusiones a Suárez y al papel que desempeñó en la configuración del escotismo desde 1600 hasta 1750 . Sin embargo, no se ha estudiado aún la genealogía del suarismo en la misma época y estamos seguros de que habría numerosos puntos de conexión y de conflicto. Lo que Andersen dice, por ejemplo, de Hurtado y de Arriaga, debería extenderse también a los tratados filosóficos iuxta mentem Suarii, escritos en el siglo XVIII, puesto que la discusión y el debate entre los epígonos continuó y al final acabó estandarizándose, con una historia cuyos entresijos aún no conocemos. En la tesis doctoral de Jacob Schmutz hay algunas intuiciones muy interesantes para dicha labor.

El segundo sería un análisis de las conexiones del escotismo con otras doctrinas escolásticas y no escolásticas en este período. El autor apunta a la obra de algunos autores más o menos eclécticos, como Lallemandet o Caramuel, que deberían ser analizadas en el diálogo que (de forma paralela) el escotismo llevaba a cabo con otras corrientes, especialmente con el nominalismo y el lulismo. El autor subraya la relevancia del nominalismo y la caracterización que de él hizo Mastri, aunque sería deseable hacer un rastreo de los intersticios del nominalismo y del escotismo en los siglos XV a XVII, así como también de los que se hallan entre el lulismo y el escotismo, especialmente tras las aportaciones de Pere Daguí, con proyecciones que llegan a autores coetáneos de Mastri, como el observante Francesc Marçal o el jesuita ecléctico Athanasius Kircher.

El tercero sería el análisis de la tradición filosófica escotista en la historia de las Universidades europeas. Hace falta conocer la interrelación entre el escotismo de los lectorados de observantes, conventuales 
y capuchinos, y el que se enseñó en las cátedras de Escoto que hubo en las diferentes universidades. Cabe preguntarse, por ejemplo, al hilo de los hallazgos de este libro, cuál fue la orientación del escotismo italiano o español (por ejemplo, las cátedras de Escoto en Alcalá, Salamanca... o las de Padua, Nápoles o La Sapienza...) ¿Se siguió el escotismo de Mastri, se continuó la opinión de los scotistae veteres o hubo sincretismo con otras corrientes?

El cuarto sería la fecha final que Andersen escoge (1750), para dar por finalizado el el "escotismo barroco". Es cierto que hacia 1750-1760, e incluso en algunos países ya antes, hay síntomas inequívocos de agotamiento y de que incluso los epígonos ya no podían absorber las tendencias del pensamiento moderno. Con todo, sería interesante definir qué tipo de escotismo (o de escotismos) hallamos en obras marginales (algunas citadas por Andersen en la p. 72, n. 48) como, por ejemplo, las de A. Winckler, Dissertatio critico-philosophica de philosophia scotistica a novatorum convitiis vindicata, una cum elementis mathematico-experimentalibus ad principia scotistica reductis, Regensburg, 1755 o las de J. A. Ferrari, que el autor cita en varios lugares más.

El predominio (y, en ocasiones, la discusión) de las tesis de Mastri se puede ver en las numerosas tesis o conclusiones escotistas sostenidas durante los siglos XVII y XVIII. Por ejemplo, Asserta philosophica ad mentem subtilissimi Scoti propugnanda, del observante Bonaventura de Politio, bajo la presidencia de Celestino Tiessen, Roma, 1673, en la que se hallan extractadas las tesis metafísicas en dos páginas y media al final de la obra, que condensan buena parte del estado estandarizado de la cuestión. O también los Theoremata antiquo-nova ex universa Aristotelis philosophia iuxta mentem Ioanni Duns Scoti doctrinam, de carácter predominantemente matafísico, defendidos en 1666 en la congregación capitular de Ingoldstadt en 1666.

Y, puestos a pedir, tras el monumental despliegue de precisión y erudición de Claus A. Andersen, sería muy estimable que él y otros escotistas elaboraran un censo completo de los textos filosóficos (manuscritos e impresos) de la tradición escotista moderna, a fin de elaborar una completa genealogía del escotismo hasta finales de la Ilustración. Con los recursos que hay actualmente en la red, sería una herramienta utilísima y permitiría afinar las variaciones conceptuales que se producen hasta el declive definitivo de la escolástica.

En fin, con este libro se completa un eslabón fundamental en la historia de la metafísica escotista, aún tan desconocida, y sobre la que falta llevar a cabo una suerte de companion o trabajo colectivo que permita conocer las fases y ramificaciones de la recepción (e incluso traición) a Escoto por parte de algunos de sus seguidores. Esperemos que Claus A. Andersen pueda proseguir su benemérita labor en algunas de estas sendas y que siga dando frutos tan excelentes como este libro.

RAFAEL RAMIS BARCELO Universitat de les Illes Balears 